Pacific Journal of Mathematic 


\title{
ON THE SINGULARITIES OF TAYLOR SERIES WITH RECIPROCAL COEFFICIENTS
}

\author{
SHMUEL AgMON
}

1. Introduction. Let

$$
f(z)=\sum_{n=0}^{\infty} a_{n} z^{n}
$$

be a Taylor series with a nonvanishing radius of convergence and such that $a_{n} \neq 0$. Put

$$
f_{-1}(z)=\sum_{n=0}^{\infty} \frac{z^{n}}{a_{n}},
$$

and suppose that the latter series also has a positive radius of convergence. Now $f_{-1}(z)$ can be considered as the inverse of $f(z)$ under the Hadamard "multiplication" of series, and it is natural to inquire into the existence of a simple relation between the singularities of the two functions. This problem was treated by Soula [3], who discovered such a relation for the singularities of the two series on their circles of convergence. His result is as follows:

THEOREM S. Let $f(z)$ and $f_{-1}(z)$ be defined by (1.1) and (1.1'), where $a_{n}$ is real, $a_{n} \neq 0$, and where, furthermore,

$$
\lim _{n \neq \infty}\left|a_{n}\right|^{1 / n}=1
$$

(Thus the unit circle is the circle of convergence for both series.) If $z=1$ is the only singularity of $f(z)$ on the unit circle then either the unit circle is a cut for $f_{-1}(z)$, or $f_{-1}(z)$ also has $z=1$ as its only singularity on the unit circle. Moreover, in the latter case we have:

(i) $\lim \left(a_{n} / a_{n+1}\right)=1$;

Received November 26, 1951.

Pacific J. Math. 2 (1952), 431-453 
(ii) $z=1$ is a singularity "without contact" (with the unit circle) for both functions $f(z)$ and $f_{-1}(z)$. That is, there exist $\delta>0$ and $\phi$, with $0<\phi<\pi / 2$, such that $f(z)$ and $f_{-1}(z)$ are analytic in the sector

$$
0<|z-1|<\delta, \phi<|\arg (z-1)| \leq \pi
$$

We remark that if the radius of convergence of $f(z)$ is 1 (which is no loss of generality) then it is not difficult to see that one cannot expect to have a dependence even between the singularities of the two series on their circles of convergence unless the radius of convergence of $f_{-1}(z)$ is also 1. Thus (1.2) is a necessary restriction. Also it seems that the condition that $f(z)$ should have only one singular point, say $z=1$, on its circle of convergence is essential for the simple character of the result. However, the condition on the reality of the coefficients in Soula's theorem is superfluous. All that is needed in Soula's proof when the coefficients are complex is the use of Lemma 5 of this paper. In what follows we shall refer to this more general result as Theorem S.

We propose in this paper to obtain a relation between the singularities of $f(z)$ and $f_{-1}(z)$ outside the unit circle. To this end it is necessary to have some information on the location of the singularities of $f(z)$ outside the unit circle. We shall impose on $f(z)$ the somewhat restrictive condition that it be holomorphic in the whole plane cut along the line $1 \leq x<\propto$. With this condition, however, we shall derive a surprisingly simple result concerning the location of the singularities of $f_{-1}(z)$ in the whole plane.

2. Preliminary considerations. We collect in this section the definitions and lemmas which we shall need in the proof of our main result. Some of these lemmas are well-known theorems.

DEFINITION. Let $f(z)$ be given in the neighborhood of the origin by the Taylor series (1.1). The star of holomorphy of $f(z)$ (Mittag-Leffler star) is defined as the domain composed of all segments $t e^{i \theta}, 0 \leq t<\rho(\theta)$, where $\rho(\theta) e^{i \theta}$ is the first singularity of $f(z)$ on the ray $t e^{i \theta}(0 \leq t<\infty)$ when $f(z)$ is continued analytically along this ray. The function $\rho(\theta)$ shall be called the star-function and shall be defined by periodicity for all values of $\theta$.

It follows readily from the definition that $\rho(\theta)$ is a lower semicontinuous function, and as such it attains its lower bound in any finite interval.

LEmma 1. (Hadamard's multiplication theorem for stars $[1, \mathrm{p} .300]$ ). Let 


$$
f(z)=\sum_{n=0}^{\infty} a_{n} z^{n} \text { and } g(z)=\sum_{n=0}^{\infty} b_{n} z^{n}
$$

have the radii of convergence $R_{f}$ and $R_{g}$, respectively, and star-functions $\rho_{f}(\theta)$ and $\rho_{g}(\theta)$. Put

$$
h(z)=[f, g]=\sum_{n=0}^{\infty} a_{n} b_{n} z^{n} .
$$

Then $h(z)$ converges for $|z|<R_{f} R_{g}$ and can be continued analytically along the segment te $e^{i \theta}, 0 \leq t<r(\theta)$, for any $0 \leq \theta<2 \pi$, where

$$
r(\theta)=\min _{0 \leq u^{<} 2 \pi} \rho_{f}(u) \rho_{g}(\theta-u) .
$$

The following is a simple lemma on the separation of singularities of an analytic function.

LEMMA 2. Let $f(z)$ be an analytic function in the neighborhood of the origin where it has the Taylor development (1.1), and let $\rho(\theta)$ be its starfunction. Then, given $\theta_{1}$ and $\theta_{2}, 0<\theta_{1}<\theta_{2}<2 \pi$, and $\epsilon>0$, there exist two analytic functions $g_{1}(z)$ and $g_{2}(z)$ with developments

$$
g_{1}(z)=\sum_{n=0}^{\infty} a_{n}^{\prime} z^{n}, g_{2}(z)=\sum_{n=0}^{\infty} a_{n}^{\prime \prime} z^{n},
$$

such that

(i) $f(z)=g_{1}(z)+g_{2}(z)$;

(ii) the star-function $\rho_{i}^{g}(\theta)$ of $g_{i}(z)$ satisfy

$$
\left\{\begin{array}{l}
\rho_{1}^{g}(\theta)=\rho(\theta) \text { and } \rho_{2}^{g}(\theta)=\infty \text { for } \theta_{1}<\theta<\theta_{2}, \\
\rho_{1}^{g}(\theta)=\infty \text { and } \rho_{2}^{g}(\theta)=\rho(\theta) \text { for } \theta_{2}<\theta<\theta_{1}+2 \pi, \\
\rho_{i}^{g}\left(\theta_{j}\right)>\rho\left(\theta_{j}\right)-\epsilon \text { for } i, j=1,2 .
\end{array}\right.
$$

We shall indicate an easy proof of Lemma 2. Let $C$ be a star-shaped rectifiable Jordan curve enclosing the origin, contained in the star of holomorphy of 
$f(z)$, and whose defining function $R=R(\theta)(0 \leq \theta<2 \pi)$ satisfies

$$
R\left(\theta_{i}\right)>\rho\left(\theta_{i}\right)-\epsilon \quad(i=1,2) .
$$

Let $C_{1}$ be the part of $C$ in the sector $\theta_{1} \leq \theta \leq \theta_{2}$, and $C_{2}$ the complementary part of $C$. Then by Cauchy's theorem we have

$$
\begin{aligned}
f(z)=\frac{1}{2 \pi i} \int_{C} \frac{f(\zeta)}{\zeta-z} d \zeta & =\frac{1}{2 \pi i} \int_{C_{1}} \frac{f(\zeta)}{\zeta-z} d \zeta+\frac{1}{2 \pi i} \int_{C_{2}} \frac{f(\zeta)}{\zeta-z} d \zeta \\
& =g_{1}(z)+g_{2}(z) .
\end{aligned}
$$

It follows now readily that the functions $g_{1}(z)$ and $g_{2}(z)$ satisfy the conditions of Lemma 2.

The following two lemmas are known. (For example, see [2, p. 103, Th. III], where the lemmas are generalized to Dirichlet series). We remark, however, that we make a trivial addition (without proof) to the lemmas by not assuming the angle in question to be symmetric with respect to the positive axis.

Lemma 3. Let

$$
f(z)=\sum_{n=0}^{\infty} a_{n} z^{n}
$$

be a Taylor series having the unit circle for its circle of convergence. Suppose that $f(z)$ can be continued analytically in a domain $D_{a_{\beta}}$ whose boundary is composed of the two spirals:

$$
\rho=\exp [(\tan \alpha) \theta] \quad\left(0 \leq \theta \leq \theta_{0}\right),
$$

and

$$
\rho=\exp [(\tan \beta)(2 \pi-\theta)] \quad\left(\theta_{0} \leq \theta \leq 2 \pi\right),
$$

where $0<\alpha, \beta<\pi / 2$ and $\theta_{0}=(2 \pi \tan \beta) /(\tan \alpha+\tan \beta)$, and where $z=\rho e^{i \theta}$. Then, given $0<\alpha^{\prime}<\alpha$ and $0<\beta^{\prime}<\beta$, there exists an "interpolation function" $G(u)$, analytic in the angle $-\beta^{\prime} \leq \arg u \leq \alpha^{\prime}$, such that

$$
G(n)=a_{n} \quad(n=0,1, \ldots),
$$




$$
\limsup _{|u|=\infty}\left(\frac{\log |G(u)|}{|u|}\right) \leq 0,
$$

uniformly in $-\beta^{\prime} \leq \arg u \leq \alpha^{\prime}$.

The next lemma is a kind of converse of Lemma 3.

LEMMA $3^{\prime}$. Let $G(u)$ be an analytic function of exponential type in the infinite sector $-\beta \leq \arg u \leq \alpha,|u| \geq R_{0}(0<\alpha, \beta<\pi / 2)$ satisfying (2.5 $)$. Let

$$
f(z)=\sum_{n>R_{0}} G(n) z^{n}
$$

Then $f(z)$ can be continued analytically in the domain $D_{a_{\beta}}$.

We shall prove now the following lemma.

Lemma 4. Let $G(u)$ be an analytic function of exponential type in the angle $-\beta \leq \arg u \leq \alpha \quad(0<\alpha, \beta<\pi / 2)$. Put

$$
\tau_{G}(\theta)=\lim _{r=\infty} \sup \left(\frac{\log \left|G\left(r e^{i \theta}\right)\right|}{r}\right) .
$$

Suppose that

$$
\left\{\begin{array}{l}
\tau_{G}(\theta) \leq \Omega_{1} \sin \theta \text { for } 0 \leq \theta \leq a, \\
\tau_{G}(\theta) \leq-\Omega_{2} \sin \theta \text { for }-\beta \leq \theta \leq 0,
\end{array}\right.
$$

where $\Omega_{1} \geq 0, \Omega_{2} \geq 0$ and $\Omega_{1}+\Omega_{2}<2 \pi$, and that

$$
G(n)=0 \text { for } n=0,1, \ldots \text {. }
$$

Let $\alpha^{*}$ and $\beta^{*}\left(0<\alpha^{*}, \beta^{*}<\pi / 2\right)$ be defined by

$$
\left\{\begin{array}{l}
\tan \alpha^{*}=\frac{2 \pi-\left(\Omega_{1}+\Omega_{2}\right)}{\Omega_{1} \cot \beta+\left(2 \pi-\Omega_{2}\right) \cot \alpha} \\
\tan \beta^{*}=\frac{2 \pi-\left(\Omega_{1}+\Omega_{2}\right)}{\left(2 \pi-\Omega_{1}\right) \cot \beta+\Omega_{2} \cot \alpha}
\end{array},\right.
$$

Then we have $\tau_{G}(\theta)<0$ for $-\beta^{*}<\theta<\alpha^{*}$, and, in particular, 


$$
G(u)=O\left(e^{-\delta|u|}\right),
$$

uniformly in any angle $-\beta^{*}<-\bar{\beta} \leq \arg u \leq \bar{\alpha}<\alpha^{*}$ for some $\delta=\delta(\bar{\alpha}, \bar{\beta})>0$.

Proof. Put

$$
F(u)=\frac{G(u)}{\sin \pi u}
$$

It is easily seen that $F(u)$ is analytic and of exponential type in the angle $-\beta \leq \arg u \leq \alpha$. Let $\tau_{F}(\theta)$ be the "type function" (2.6) of $F(u)$. It follows from (i) and (2.9) that

$$
\tau_{F}(\alpha) \leq\left(\Omega_{1}-\pi\right) \sin \alpha \text { and } \tau_{F}(-\beta) \leq\left(\Omega_{2}-\pi\right) \sin \beta .
$$

Applying a weli-known result of Phragmén and Lindelöf [5, p.183], we deduce from the last two inequalities that

$$
\tau_{F}(\theta) \leq A \cos \theta+B \sin \theta,
$$

where $A$ and $B$ are the solutions of

$$
\begin{aligned}
& A \cos \alpha+B \sin \alpha=\left(\Omega_{1}-\pi\right) \sin \alpha, \\
& A \cos \beta-B \sin \beta=\left(\Omega_{2}-\pi\right) \sin \beta .
\end{aligned}
$$

That is:

$$
A=\frac{\Omega_{1}+\Omega_{2}-2 \pi}{\cot \alpha+\cot \beta}, B=\frac{\left(\Omega_{1}-\pi\right) \cot \beta-\left(\Omega_{2}-\pi\right) \cot \alpha}{\cot \alpha+\cot \beta} .
$$

Hence, from (2.9), (2.10), and $\left(2.10^{\circ}\right)$, we get

$$
\tau_{G}(\theta) \leq A \cos \theta+B \sin \theta+\pi|\sin \theta| \quad(-\beta \leq \theta \leq \alpha) .
$$

The assertion of the lemma now follows from (2.11) if we note that the right side of $(2.11)$ is a continuous negative function for $-\beta^{*}<\theta<\alpha^{*}$. (We also make use of the well-known fact that an analytic function of exponential type satisfying (2.11) also satisfies

$$
G(u)=O(\exp [|u|(A \cos \theta+(B+\pi) \sin \theta+\epsilon)])
$$

uniformly in the angle.) 
Finally, we shall need the following lemma which is a generalization of a lemma due to Soula [3, p.38]. It is stated in a somewhat more general form than is needed for our purpose here; however, the lemma in this form is required for the completion of Soula's theorem (Theorem S) mentioned in 1 .

Lemma 5. Let $h(z)$ be an analytic function in the infinite sector $|z| \geq R_{0}$, $|\arg z| \leq \alpha<\pi / 2$, where it satisfies

$$
\lim _{n=\infty} \frac{R\{h(n)\}}{n}=0
$$

( $n$ being an integer);

(ii) there exists a nonnegative, continuous, and increasing function $\delta(\theta)$, $0 \leq 0 \leq \alpha$, with $\delta(0)=0$, such that

$$
R\left\{h\left(r e^{i \theta}\right)\right\}=[\delta(|\theta|)|\sin \theta|+\epsilon] r,
$$

for any $\epsilon>0, r \geq r_{0}(\epsilon)$ large enough, and $|\theta| \leq a$. Then

$$
\lim _{|z| \rightarrow \infty}\left(\frac{h(z)}{z}\right)=0,
$$

uniformly in any sector $|\arg z| \leq a^{\prime}<\alpha,|z| \geq k_{0}$.

Proof. We shall make use of the well-known inequality

$$
|f(z)-f(0)| \leq \frac{2|z|(A(R)-A(0))}{R-|z|},
$$

where $f(z)$ is analytic for $|z| \leq R$, and

$$
A(R)=\max _{|z|=R} P_{j}\{f(z)\} .
$$

We shall first show that

$$
\lim _{n=\infty}\{h(n+b-h(n)\}=0
$$

uniformly for $\zeta$ in any bounded set. Indeed, choose $\eta_{\text {i }}$ such that $0<\eta<c_{\text {, }}$, and let $|\zeta| \leq C$. Let $n+z=r e^{i \theta}$ be a point inside the circle of radius $R=n \sin \eta$ and center $z=n$ inscribed inside the angle $|\arg z| \leq \eta$. On account of (ii), taking $\epsilon=\delta(\eta) \sin \eta$ and $n$ large enough, we have 


$$
\begin{aligned}
R\left\{h\left(r e^{i \theta}\right)\right\} & \leq 2 \delta(\eta) \sin \eta r \leq 2 \delta(\eta) \sin \eta(1+\sin \eta) n \\
& \leq(4 \delta(\eta) \sin \eta) n .
\end{aligned}
$$

Applying (2.13) to $f(z)=h(n+z)$ for $z=\zeta, R=n \sin \eta$, and using (2.15), we get

$$
\begin{aligned}
|h(n+\zeta)-h(n)| & \leq \frac{2|\zeta|[(4 \delta(\eta) \sin \eta) n+|R\{h(n)\}|]}{n \sin \eta-|\zeta|} \\
& \leq \frac{8 C \sin \eta \delta(\eta)}{\sin \eta-C / n}+\frac{2 C}{\sin \eta-C / n}\left|R\left\{\frac{h(n)}{n}\right\}\right| .
\end{aligned}
$$

Sending $n$ to infinity and using (i), we find that

$$
\lim \sup |h(n+\zeta)-h(n)| \leq 8 C \delta(\eta)
$$

uniformly for $|\zeta| \leq C$. Letting $\eta$ tend to zero, and recalling that $\delta(0)=0$, we arrive at $(2.14)$. In what follows we shali need only the weaker result

$$
\lim _{x \rightarrow \infty}\left(\frac{h(x)}{x}\right)=0
$$

which follows in an obvious way from (2.14).

We next show that $h(z) / z$ is bounded in every sector

$$
|\arg z| \leq \alpha^{\prime}<\alpha,|z| \geq R_{0}
$$

This will be established if we prove that $h(z) / z$ is bounded uniformly in the circles $C_{\xi}:|z-\xi| \leq \xi \sin \alpha^{\prime}(\xi$ large enough). Now, as before, for $\xi$ positive and large enough, and for $z$ such that $|z| \leq \xi \sin \alpha$, we have

$$
\text { R. }\{h(\xi+z)\} \leq(4 \delta(\alpha) \sin \alpha) \xi \text {. }
$$

Applying (2.13), for $|z| \leq \xi \sin \alpha^{\prime}$ we find

$$
|h(\xi+z)-h(\xi)| \leq \frac{8 \delta(\alpha) \sin \alpha^{\prime} \sin \alpha}{\sin \alpha-\sin \alpha^{\prime}} \xi+\frac{2 \sin \alpha^{\prime}}{\sin \alpha-\sin \alpha^{\prime}}|R\{h(\xi)\}|,
$$

from which we get that

$$
|h(\xi+z)| \leq C_{1} \xi+C_{2}|h(\xi)|,
$$


where $C_{1}$ and $C_{2}$ are constants depending only on $\alpha$ and $\alpha^{\prime}$. We have only to divide (2.17) by $\xi+z$ and use (2.16) in order to obtain the uniform boundedness of $h(\xi+z) /(\xi+z)$ for $|z| \leq \xi \sin \alpha^{\prime}(\xi \rightarrow \infty)$, and therefore the boundedness of $h(z) / z$ in any interior sector. To complete the lemma we apply a well-known result of Phragmen and Lindelof by which the boundedness and (2.16) imply (2.12). We also note that by successive applications of the last lemma it follows that the result still holds if the angle containing the positive axis is not supposed to be symmetric.

3. The main theorem. We pass now to our main result:

THEOREM 1. Let $f(z)$ be an analytic function in the whole plane cut along the line $1 \leq x<\infty$. Suppose that the coefficients of its Taylor expansion (1.1) are different from zero and satisfy (1.2). Let $f_{-1}(z)$ be the "inverse" series defined by $\left(1.1^{\prime}\right)$, and denote by $\rho_{-1}(\theta)$ its star-function (Def. $\$ 2$ ). Then there exist two numbers $\alpha, \beta$, with $0 \leq \alpha \leq \pi / 2$ and $0 \leq \beta \leq \pi / 2$, such that for any $0<\theta<2 \pi$ we have

$$
\rho_{-1}(\theta)=\min \left\{e^{(\tan \alpha) \theta}, e^{(\tan R)(2 \pi-\theta)}\right\},
$$

and (trivially) $\rho_{-1}(0)=1$.

The theorem states, in other words, that the star of holomorphy of $f_{-1}(z)$ consists in general of the two logarithmic spirals:

$$
\rho_{-1}(\theta)=\exp \left[\left(\tan \alpha_{1}\right) \theta\right] \text { for } 0 \leq \theta \leq \psi,
$$

and

$$
\rho_{-1}(\theta)=\exp [(\tan \beta)(2 \pi-\theta)] \text { for } \psi \leq \theta \leq 2 \pi \text {, }
$$

where $\psi$ is given by

$$
\psi=\frac{2 \pi \tan \beta}{\tan \alpha+\tan \beta}
$$

(We shall treat $\theta=0$ separately in order to account for the case where either $\alpha$ or $\beta$ is equal to $\pi / 2$, in which case we agree that the product of a positive number and infinity is infinity.)

It is possible to distinguish the following cases which correspond to limiting values of $\alpha$ and $\beta$ : (i) $\alpha=0$ or $\beta=0$. In this case we have $\rho_{-1}(\theta) \equiv 1$, and the 
unit circle is a cut for $f_{-1}(z)$; this is a particular case of Theorem S.

(ii) $0<\alpha<\pi / 2$ and $0<\beta<\pi / 2$. In this case the star of holomorphy of $f_{-1}(z)$ is the domain $D_{\alpha \beta}$ (introduced in Lemma 3 ), whose boundary consists of the two spirals $\left(3.1^{\prime}\right)$; this domain is also the region of existence of $f_{-1}(z)$.

(iii) $0<\alpha<\pi / 2$ while $\beta=\pi / 2$. In this case all the points of the spiral $\rho=\exp [(\tan \alpha) \theta] \quad(0 \leq \theta<2 \pi)$ are singularities. (However, this does not exclude the possibility of analytic continuation through the segment $1<x<$ $\exp [(\tan a) 2 \pi]$.

(iv) $\alpha=\pi / 2$ while $0<\beta<\pi / 2$. This case is similar to the preceding one; the only difference is that the roles of $\alpha$ and $\beta$ are interchanged.

(v) $\alpha=\beta=\pi / 2$. In this case $f_{-1}(z)$ has the properties of $f(z)$, and is analytic in the whole plane cut along $1 \leq x<\infty$.

Proof of Theorem 1. Since $f(z)$ satisfies the conditions of Theorem S, it follows that our results will go beyond those of Soula only when $z=1$ is the only singularity of $f_{-1}(z)$ on the unit circle, and when it is, furthermore, "without contact". We shall assume in what follows that this is indeed the case. Now, the proof of the theorem is somewhat long and will be divided into two parts.

Part I. Let us define $\alpha, \beta$, with $0<\alpha \leq \pi / 2$ and $0<\beta \leq \pi / 2$, by the relations

$$
\left\{\begin{array}{l}
\tan c_{i}=\limsup _{h \rightarrow+0} \frac{\log \rho_{-1}(h)}{h}, \\
\tan \beta=\limsup _{h \rightarrow+0} \frac{\log \rho_{-1}(-h)}{h} .
\end{array}\right.
$$

We shall establish in this part of the proof that:

(a) The interval $0 \leq \theta \leq 2 \pi$ can be divided into two disjoint intervals $I_{1}$ and $I_{2}$, where $I_{1}$ is the interval $0 \leq \theta<\omega$ or $0 \leq \theta \leq \omega$, and $I_{2}$ is the interval $\omega \leq \theta \leq 2 \pi$ or $\omega<\theta \leq 2 \pi$ (one of the intervals can consist of a single point), such that $\rho_{-1}(\theta)$ is increasing in $I_{1}$ and decreasing in $I_{2}$, and such that furthermore, the equality $\rho_{-1}\left(\theta_{1}\right)=\rho_{-1}\left(\theta_{2}\right)$ for $\theta_{1}<\theta_{2}, \theta_{1}$ and $\theta_{2}$ both in to the same interval, can hold only if $\rho_{-1}(\theta) \equiv \infty$ for $\theta_{1} \leq \theta \leq \theta_{2}$.

(b) The following inequalities hold: 


$$
\left\{\begin{array}{l}
\log \rho_{-1}(\theta) \geq(\tan \alpha) \theta \text { in } I_{1}, \\
\log \rho_{-1}(\theta) \geq(\tan \beta)(2 \pi-\theta) \text { in } l_{2} .
\end{array}\right.
$$

Now, both (a) and (b) are consequences of the following inequality which we shall establish later:

$$
\rho_{-1}(\theta) \geq \min \left\{\rho_{-1}\left(\theta_{1}\right) \rho_{-1}\left(\theta-\theta_{1}\right), \rho_{-1}\left(\theta_{2}\right) \rho_{-1}\left(\theta-\theta_{2}\right)\right\},
$$

where $0<\theta_{1}<\theta<\theta_{2}<2 \pi$. Indeed, it is easily seen that (a) will be proved if we can show that the minimum of $\rho_{-1}(\theta)$ in an interval $0<\theta_{1} \leq \theta \leq \theta_{2}<2 \pi$ is attained only at one of the end-points. To establish this let us assume, by way of contradiction, that the minimum is attained at a certain inner point $\bar{\theta}$. Using now (3.4) for $\theta=\bar{\theta}$, and using the fact that both $\rho_{-1}\left(\bar{\theta}-\theta_{1}\right)$ and $\rho_{-1}\left(\theta_{2}-\right.$ $\bar{\theta})$ are greater than one, we get the absurdity

$$
\rho_{-1}(\bar{\theta})>\min \left\{\rho_{-1}\left(\theta_{1}\right), \rho_{-1}\left(\theta_{2}\right)\right\} \geq \min _{\theta_{1} \leq \theta \leq \theta_{2}} \rho_{-1}(\theta)=\rho_{-1}(\bar{\theta}) .
$$

This proves (a). We note also that from (a) follows that the points where $\rho_{-1}(\theta)=\infty$ constitute one interval.

Let us now establish (b). We shall limit ourselves to proving the first inequality; the second will follow in a similar fashion. It is clear from the above that it is enough to prove the first inequality (3.3) for $\theta$ which is interior to $I_{1}$ and such that $\rho_{-1}(x)$ is finite for $0 \leq x \leq \theta+\epsilon, \epsilon$ being a small positive number depending on $\theta$ (if no such $\theta$ exists, then $\rho_{-1}(\theta) \equiv \infty$ for $\theta \in I_{1}, \theta \neq 0$, and the inequality is satisfied with $\alpha=\pi / 2)$. Let now $h>0$ be such that $\theta+$ $2 h \in l_{1}$, and let us apply (3.4) to $\theta_{1}(=) \theta, \theta(=) \theta+h$ and $\theta_{2}(=) \theta+2 h$. We get

$$
\rho_{-1}(\theta+h) \geq \min \left\{\rho_{-1}(\theta) \rho_{-1}(h), \rho_{-1}(\theta+2 h) \rho_{-1}(-h)\right\}
$$

Since $\rho_{-1}(-h)>1$ and $\rho_{-1}(\theta+2 h) \geq \rho_{-1}(\theta+h)$, we obviously must have

$$
\rho_{-1}(\theta+h) \geq \rho_{-1}(\theta) \rho_{-1}(h) \text {. }
$$

Passing to logarithms, from the last inequality we get

$$
\limsup _{h \rightarrow+0} \frac{\log \rho_{-1}(\theta+h)-\log \rho_{-1}(\theta)}{h} \geq \tan \alpha,
$$


where $\alpha$ was defined by (3.2). But, since $\log \rho_{-1}(\theta)$ is an increasing function in $I_{1}$, its derivative exists almost everywhere in $I_{1}^{*}$, the largest sub-interval of $I_{1}$ where $\rho_{-1}(\theta)$ is finite. Making use of $(3.5)$, we see that almost everywhere in $I_{1}^{*}$, we have

$$
\left[\log \rho_{-1}(\theta)\right]^{\prime} \geq \tan \alpha
$$

We now employ a simple inequality applicable to any nondecreasing function $g(x)[5$, pp. 361, 373]:

$$
g(b)-g(a) \geq \int_{a}^{b} g^{\prime}(x) d x,
$$

where the integral is taken in the Lebesgue sense. When applying this to the function $\log \rho_{-1}(\theta)-(\tan \alpha) \theta$ in $\left[\theta_{1}, \theta_{2}\right]$, using (3.6), we conclude that $\log \rho_{-1}(\theta)-(\tan \alpha) \theta$ is nondecreasing in $I_{1}^{*}$, and a fortiori in the interval $I_{1}$. The desired first inequality (3.3) now follows if we note that $\log \rho_{-1}(\theta)-$ $(\tan \alpha) \theta$ vanishes for $\theta=0$.

W'e still have to establish (3.4). Let $\theta_{1}$ and $\theta_{2}$ satisfy $0<\theta_{1}<\theta_{2}<2 \pi$, and let $\epsilon$ be an arbitrary positive number. By Lemma 2 there exist two analytic functions $g_{1}(z)$ and $g_{2}(z)$, with star-functions $\rho_{1}^{g}(\theta)$ and $\rho_{2}^{g}(\theta)$, such that

$$
f_{-1}(z)=g_{1}(z)+g_{2}(z),
$$

and such that the corresponding star-functions satisfy the relations (2.3). Now the Hadamard multiplication of the two "inverse" functions $f(z)$ and $f_{-1}(z)$ is the "unit" function $1 /(1-z)$, so that if we define

$$
h_{1}(z)=\left[f(z), g_{1}(z)\right] \text { and } h_{2}(z)=\left[f(z), g_{2}(z)\right] \text {, }
$$

we get

$$
\begin{aligned}
\frac{1}{1-z} & =\left[f(z), f_{-1}(z)\right]=\left[f(z), g_{1}(z)+g_{2}(z)\right] \\
& =\left[f(z), g_{1}(z)\right]+\left[f(z), g_{2}(z)\right]=h_{1}(z)+h_{2}(z) .
\end{aligned}
$$

Let us now denote by $\rho_{1}^{h}(\theta)$ and $\rho_{2}^{h}(\theta)$ the star-functions of $h_{1}(z)$ and $h_{2}(z)$. It follows again from (3.7) and Hadamard's multiplication theorem, since $f(z)$ 
is analytic in the whole plane cut along $1 \leq x<\infty$, that

$$
\rho_{1}^{h}(\theta) \geq \rho_{1}^{g}(\theta) \text { and } \rho_{2}^{h}(\theta) \geq \rho_{2}^{g}(\theta) .
$$

Using properties (2.3) of $\rho_{i}^{g}(\theta)$, we see that

$$
\left\{\begin{array}{l}
\rho_{1}^{h}(\theta) \equiv \infty \text { for } \theta_{2}<\theta<\theta_{1}+2 \pi, \\
\rho_{2}^{h}(\theta) \equiv \infty \text { for } \theta_{1}<\theta<\theta_{2} .
\end{array}\right.
$$

(In other words, $h_{1}(z)$ and $h_{2}(z)$ are analytic in the angles $\theta_{2}<\arg z<\theta_{1}+$ $2 \pi$ and $\theta_{1}<\arg z<\theta_{2}$ respectively.) We also find that

$$
\rho_{i}^{h}\left(\theta_{j}\right) \geq \rho_{-1}\left(\theta_{j}\right)-\epsilon \quad(i, j=1,2) .
$$

Moreover, since $h_{1}(z)$ and $h_{2}(z)$ add to $1 /(1-z)$, a function having its only singularity at $z=1$, we conclude that

$$
\rho_{\mathbf{1}}^{h}(\theta) \equiv \infty \text { for } \theta_{1}<\theta<\theta_{2}
$$

Let us consider now the Hadamard multiplication of $f_{-1}(z)$ and $h_{1}(z)$. Clearly, we have

$$
\begin{aligned}
{\left[f_{-1}(z), h_{1}(z)\right] } & =\left[f_{-1}(z),\left[f(z), g_{1}(z)\right]\right] \\
& =\left[\left[f_{-1}(z), f(z)\right], g_{1}(z)\right]=\left[\frac{1}{1-z}, g_{1}(z)\right]=g_{1}(z) .
\end{aligned}
$$

Using once more Hadamard's theorem, taking into account (3.9)-(3.12) for $\rho_{1}^{h}(\theta)$, and also remembering that $\rho_{1}^{g}(\theta)=\rho_{-1}(\theta)$ for $\theta_{1}<\theta<\theta_{2}$, we obtain

$$
\begin{aligned}
\rho_{-1}(\theta)=\rho_{1}^{g}(\theta) & \geq \min \left\{\rho_{1}^{h}\left(\theta_{1}\right) \rho_{-1}\left(\theta-\theta_{1}\right), \rho_{1}^{h}\left(\theta_{2}\right) \rho_{-1}\left(\theta-\theta_{2}\right)\right\} \\
& \geq \min \left\{\left(\rho_{-1}\left(\theta_{1}\right)-\epsilon\right) \rho_{-1}\left(\theta-\theta_{1}\right),\left(\rho_{-1}\left(\theta_{2}\right)-\epsilon\right) \rho_{-1}\left(\theta-\theta_{2}\right)\right\} .
\end{aligned}
$$

We now have only to send $\epsilon$ in the last inequality to zero in order to arrive at the desired result (3.4).

Part II. It follows from Part I that if $\alpha$ and $\beta$ are defined by (3.2), then

$$
\log \rho_{-1}(\theta) \geq \min \{(\tan \alpha) \theta,(\tan \beta)(2 \pi-\theta)\} .
$$


Theorem 1 will, therefore, be established if we show that (3.13) is actually an equality. Now, we may assume that $\alpha$ and $\beta$ are not both equal to $\pi / 2$. For, if this were the case, then we would have $\rho_{-1}(\theta) \equiv \infty$ for $0<\theta<2 \pi$, and the theorem would be proved. In what follows we shall assume that the theorem is false and that $(3.13)$ is a strict inequality for a certain $\theta=\theta_{0}$. This we shall show will lead to a contradiction. Now, from the lower semicontinuity of $\rho_{-1}(\theta)$ it follows that if we do not have everywhere equality in (3.13), then for infinitely many points we have a strict inequality. This allows us to assume that $\theta_{0}$ differs from $\psi$, where $\psi$ is defined by $\left(3.1^{\prime}\right)$. There is also no loss of generality in assuming $0<\theta_{0}<\psi$, since otherwise we have only to replace $f(z)$ and $f_{-1}(z)$ by $\overline{f(\bar{z})}$ and $\overline{f_{-1}(\bar{z})}$, respectively. We first note that in the interval $[0, \psi)$

$$
(\tan \alpha) \theta<(\tan \beta)(2 \pi-\theta),
$$

and hence there exists $\delta>0$ small enough that

$$
\log \rho_{-1}\left(\theta_{0}\right) \geq(\tan \alpha) \theta_{0}+\delta
$$

We shall now define the domain $D=D\left(\alpha, \beta, \theta_{0}, \delta\right)$ as the set of points $z=r e^{i \theta}$ satisfying

$$
\left\{\begin{array}{l}
\log r<(\tan \alpha) \theta \text { for } 0 \leq \theta \leq \theta_{0}, \\
\log r<\min \{(\tan \alpha) \theta+\delta,(\tan \beta)(2 \pi-\theta)\} \text { for } \theta_{0}<\theta<2 \pi .
\end{array}\right.
$$

Let us denote by $R(\theta)$ the star-function corresponding to the boundary of $D$. We claim that

$$
R(\theta) \leq \rho_{-1}(\theta)
$$

Indeed, this is obvious from the definition of $D$ and from (3.13) so long as $0 \leq \theta \leq \theta_{0}$. If $\theta_{0}<\theta<2 \pi$, then we have to distinguish between two cases:

(i) $\theta$ belongs to the interval $I_{1}$ introduced in Part $\mathrm{I}$; then both $\theta_{0}$ and $\theta\left(\theta_{0}<\right.$ $\theta$ ) belong to $I_{1}$, and since we have established before that $\log \rho_{-1}(\theta)-(\tan \alpha) \theta$ is an increasing function in $l_{1}$, we have

$$
\log \rho_{-1}(\theta)-\left(\tan \alpha_{0}\right) \theta \geq \log \rho_{-1}\left(\theta_{0}\right)-(\tan \alpha) \theta_{0} \geq \delta,
$$




$$
\log \rho_{-1}(\theta) \geq(\tan \alpha) \theta+\delta \geq \log R(\theta)
$$

(ii) $\theta \in I_{2}$. In this case we see from (3.14) and (3.3) that

$$
R(\theta) \leq \exp [(\tan \beta)(2 \pi-\theta)] \leq \rho_{-1}(\theta) .
$$

This establishes (3.15).

Let now $\beta^{\prime}, \theta^{\prime}, \delta^{\prime}$ be such that $0<\beta^{\prime}<\beta, \theta_{0}<\theta^{\prime}<\psi^{\prime}$ and $0<\delta^{\prime}<\delta$, where $\beta^{\prime}, \theta^{\prime}$, and $\delta^{\prime}$ are chosen so near to $\beta, \theta_{0}$, and zero, respectively, that

$$
\left(\tan \beta^{\prime}\right)\left(2 \pi-\theta^{\prime}\right)>(\tan \alpha) \theta^{\prime}+\delta^{\prime} .
$$

Let $\epsilon$ and $\epsilon_{1}$ be two small positive numbers, and let

$$
D^{*}=D^{*}\left(\alpha-\epsilon, \beta^{\prime}, \theta^{\prime}, \delta^{\prime}, \epsilon_{1}\right)
$$

be the domain defined by:

(3.17) $\left\{\begin{array}{l}\log r<[\tan (\alpha-\epsilon)] \theta \text { for } 0 \leq \theta \leq \theta^{\prime}, \\ \log r<\min \left\{[\tan (\alpha-\epsilon)] \theta+\delta^{\prime},\left(\tan \beta^{\prime}\right)(2 \pi-\theta)\right\} \text { for } \theta^{\prime} \leq \theta<2 \pi, \\ \log |z-1|>\epsilon_{1} .\end{array}\right.$

Because of $(3.15)$ it is clear that $f_{-1}(z)$ is analytic in the closure of $D^{*}$. Let $C^{*}$ be the boundary of $I^{*}$, and set

$$
G(u)=\frac{1}{2 \pi i} \int_{C^{*}} f_{-1}(z) e^{-(\log z) u} \frac{d z}{z},
$$

where the determination of $\log z$ is chosen in the following manner: let $\Phi$ be the argument corresponding, to the vertex $V^{\prime}$ of $C^{*}$ where the two spirals

$$
\log r=[\tan (\alpha-\epsilon)] \theta+\delta^{\prime} \text { and } \log r=(\tan \beta)(2 \pi-\theta)
$$

intersect, that is

$$
\Phi=\frac{2 \pi \tan \beta^{\circ}-\delta^{\prime}}{\tan (\alpha-\epsilon)+\tan \beta^{\prime}}
$$

Then we choose $-(2 \pi-\Phi) \leq \arg z \leq \Phi$. It is readily seen that $G(u)$ is an entire 
function of exponential type. Furthermore, for $\epsilon_{1}$ small enough, $G(u)$ is independent of $\epsilon_{1}$. That is, if we change only $\epsilon_{1}$ and leave the other parameters in the definition of $C^{*}$ fixed, then the value of the integral (3.18) will remain unchanged. This follows easily from Cauchy's theorem if we note that the curves so obtained all have the same vertex $V$. Finally, if $n$ is a nonnegative integer, then from (3.18) and (1.1') we obtain

$$
G(n)=\frac{1}{a_{n}} .
$$

We shall now study the growth of $G(u)$ more closely. For this purpose let us put

$$
u=|u| e^{i \phi}
$$

and

$$
\Lambda_{\phi}(z)=(\arg z) \sin \phi-(\log |z|) \cos \phi
$$

From (3.18) we get

$$
\log |G(u)| \leq|u| \max _{z \in C^{*}} \Lambda_{\phi}(z)+K,
$$

where $K$ is a constant.

Now, the curve $C^{*}$ is composed of five analytic arcs,

$$
C^{*}=\sum_{i=1}^{5} C_{i}
$$

on each of which we shall evaluate the maximum of $\Lambda_{\phi}(z)$.

(i) On $C_{1}$, the arc of circle

$$
|z-1|=\epsilon_{1}, \eta_{1} \leq \arg z \leq \eta_{2},
$$

since $\eta_{1}$ and $\eta_{2}$ tend to zero with $\epsilon_{1}$, we have

$$
\begin{aligned}
\left|\Lambda_{\phi}(z)\right| & \leq|\arg z||\sin \phi|+|\log | z|||\cos \phi| \\
& \leq 2 \pi \max \left(\eta_{1}, \eta_{2}\right)+\log \left(1+\epsilon_{1}\right)=\eta_{3},
\end{aligned}
$$


where $\eta_{3}$ tends to zero with $\epsilon_{1}$.

(ii) On $C_{2}$, the spiral arc

$$
\log |z|=(\arg z) \tan (\alpha-\epsilon) \text { for } \eta_{2} \leq \arg z \leq \theta^{\prime},
$$

we have

$$
\Lambda_{\phi}(z)=(\arg z) \sin \phi(1-\cot \phi \tan (\alpha-\epsilon)) .
$$

Hence

$$
\max _{z \in C_{2}} \Lambda_{\phi}(z) \leq 0 \text { for }-\frac{\pi}{2} \leq \phi \leq 0 \text {. }
$$

On the other hand, if $0<\phi^{*}<\pi / 2$ is defined by

$$
\tan \phi^{*}=\tan (\alpha-\epsilon)+\frac{\delta^{\prime}}{\Phi}=\tan (\alpha-\epsilon)+\mu^{*},
$$

we get from (3.23) and (3.25), for $0 \leq \phi \leq \phi^{*}$,

$$
\begin{aligned}
\max _{z \in C_{2}} \Lambda_{\phi}(z) & \leq \theta^{\prime} \sin \phi\left(1-\cot \phi^{*} \tan (\alpha-\epsilon)\right. \\
& =\theta^{\prime} \sin \phi\left(1-\frac{\tan (\alpha-\epsilon)}{\tan (\alpha-\epsilon)+\mu^{*}}\right)=\Omega^{*} \sin \phi,
\end{aligned}
$$

where we put

$$
\Omega^{*}=\frac{\theta^{\prime} \mu^{*}}{\tan (\alpha-\epsilon)+\mu^{*}} .
$$

(iii) $\mathrm{On}_{3} C_{3}$, the segment

$$
\exp \left[i \theta^{\prime} t\right] \text {, where } \theta^{\prime} \tan (\alpha-\epsilon) \leq t \leq \theta^{\prime} \tan (\alpha-\epsilon)+\delta^{\prime}
$$

we obtain the same inequalities as in the preceding case.

(iv) $\mathrm{On}_{4}$, the spiral

$$
\log |z|=\delta^{\prime}+(\arg z) \tan (\alpha-\epsilon), \theta^{\prime} \leq \arg z \leq \Phi,
$$

we have 


$$
\Lambda_{\phi}(z)=(\arg z) \sin \phi\left(1-\cot \phi\left[\tan (\alpha-\epsilon)+\frac{\delta^{\prime}}{\arg z}\right]\right)
$$

from which, using ( 3.25$)$, we get

$$
\max _{z \in C_{4}} \Lambda_{\phi}(z) \leq 0 \text { for }-\frac{\pi}{2} \leq \phi \leq \phi^{*}
$$

(v) Finally, on $C_{5}$, the spiral

$$
\log |z|=-(\arg z) \tan \beta^{\prime},-(2 \pi-\Phi) \leq \arg z \leq-\eta_{1},
$$

we have

$$
\Lambda_{\phi}(z)=(\arg z) \sin \phi\left(1+\cot \phi \tan \beta^{\prime}\right) \text {, }
$$

from which it follows that

$$
\max _{z \in C_{5}} \Lambda_{\phi}(z) \leq 0 \text { for }-\beta^{\prime} \leq \phi \leq \pi / 2
$$

By combining the inequalities $(3.22)-(3.29)$, we conclude that

$$
\left\{\begin{array}{l}
\max _{z \in C^{*}} \Lambda_{\phi}(z) \leq \max \left(\eta_{3}, \Omega^{*} \sin \phi\right) \text { for } 0 \leq \phi \leq \phi^{*} \\
\max _{z \in C^{*}} \Lambda_{\phi}(z) \leq \eta_{3} \text { for }-\beta^{\prime} \leq \phi \leq 0
\end{array}\right.
$$

But $\eta_{3}$ tends to zero with $\epsilon_{1}$, while $\Omega^{*}$ and $G(z)$ are independent of $\epsilon_{1}$. This, with (3.30) and (3.21), implies that the type-function (2.6) of $G(u)$ satisfies

$$
\left\{\begin{array}{l}
\tau_{G}(\phi) \leq \Omega^{*} \sin \phi \text { for } 0 \leq \phi \leq \phi^{*}, \\
\tau_{G}(\phi) \leq 0 \text { for }-\beta^{\prime} \leq \phi \leq 0 .
\end{array}\right.
$$

Now, let $\gamma$ be a number such that $\max \left\{\alpha-\epsilon, \beta^{\prime}\right\}<\gamma<\pi / 2$. Since $f(z)$ is analytic in the whole plane cut along the half-line $1 \leq x<\infty$, there exists by Lemma 3 an analytic function $F(u)$ of exponential type in the angle $|\arg u| \leq$ $\gamma$, such that

$$
F(n)=a_{n}
$$$$
(n=0,1, \cdots) \text {, }
$$ 
and

$$
\tau_{F}(\phi) \leq 0
$$

Let us put

$$
H(u)=F(u) G(u)-1
$$

Then $H(u)$ is analytic and of exponential type in the angle $-\beta^{\prime} \leq$ arg $u \leq \phi^{*}$. Furthermore, because of $(3.31)-(3.34)$, the following relations hold:

$$
H(n)=0
$$$$
(n=0,1, \cdots)
$$

$$
\tau_{H}(\phi) \leq \Omega^{*} \sin \phi \text { for } 0 \leq \phi \leq \phi^{*}
$$

and

$$
\tau_{H}(\phi) \leq 0 \text { for }-\beta^{\prime} \leq \phi \leq 0
$$

Thus $H(u)$ satisfies the conditions of Lemma 4 with $\Omega_{1}=\Omega^{*}, \Omega_{2}=0, \alpha=\phi^{*}$ and $\beta=\beta^{\prime}$. Applying this lemma, we conclude that $H(u)$ tends uniformly to zero in any angle interior to the angle $A_{\alpha^{*} \beta^{*}}:-\beta^{*}<\arg u<\alpha^{*}$, where $\alpha^{*}$ and $\beta^{*}\left(0<\alpha^{*}<\pi / 2,0<\beta^{*}<\pi / 2\right)$ are defined by

$$
\left\{\begin{array}{l}
\tan \alpha^{*}=\frac{2 \pi-\Omega^{*}}{\Omega^{*} \cot \beta^{\prime}+2 \pi \cot \phi^{*}}, \\
\tan \beta^{*}=\frac{2 \pi-\Omega^{*}}{\left(2 \pi-\Omega^{*}\right) \cot \beta^{\prime}}=\tan \beta^{\prime} .
\end{array}\right.
$$

From the last property, and from (3.34), it follows in particular that $F(u)$ can have only a finite number of zeros in any angle interior to $A_{\alpha^{*} \beta^{*}}$. Let now $\bar{\alpha}$, $\bar{\beta}$ satisfy $0<\bar{\alpha}<a^{*}$ and $\theta<\bar{\beta}<\beta^{*}$, and let $R_{0}$ be large enough so that $F(u) \neq 0$ in the sector

$$
\Sigma_{\bar{\alpha}, \bar{\beta}, R_{0}}:-\bar{\beta} \leq \arg u \leq \bar{\alpha},|u| \geq R_{0}
$$

In this sector any determination of $\log F(u)$ is analytic and satisfies, because of (3.32), (3.33), and (1.2), the conditions of Lemma 5. Hence 


$$
\lim \frac{\log |F(u)|}{|u|}=0
$$

uniformly in any sector interior to $\Sigma \bar{a}, \bar{\beta}, R_{0}$. Let us put $F_{-1}(u)=1 / F(u)$. Then $F_{-1}(u)$ is analytic in $\Sigma \bar{\alpha}, \bar{\beta}_{0} R_{0}$ and satisfies

$$
F_{-1}(n)=\frac{1}{a_{n}} \text { for } n>R_{0},
$$

$$
\lim _{|u| \rightarrow \infty} \frac{\log \left|F_{-1}(u)\right|}{|u|}=0,
$$

uniformly in any sector interior to $\Sigma_{\bar{a}, \bar{\beta}, R_{0}}$. We can now apply Lemma $3^{\prime}$, and conclude that

$$
\sum_{n>R_{0}} F_{-1}(n) z^{n}=\sum_{n>R_{0}} \frac{z^{n}}{a_{n}}
$$

is analytic in the domain $D_{\bar{\alpha}}, \bar{\beta}$ bounded by the two spirals

$$
r=\exp [(\tan \bar{\alpha}) \theta] \text { for } 0 \leq \theta \leq \bar{\psi},
$$

and

$$
r=\exp [(\tan \beta)(2 \pi-\theta)] \text { for } \bar{\psi} \leq \theta \leq 2 \pi,
$$

where $\bar{\psi}$ is the expression $\left(3.1^{\prime}\right)$ with bars. Obviously the function $f_{-1}(z)$ will be analytic in the same region. Moreover, since $\bar{\alpha}$ and $\bar{\beta}$ can be chosen as near as we please to $\alpha^{*}$ and $\beta^{*}$, respectively, it follows that $f_{-1}(z)$ is analytic in $D_{\alpha^{*}, \beta^{*}}$. This gives us, in particular, the inequality

$$
\log \rho_{-1}(\theta) \geq\left(\tan \alpha^{*}\right) \theta \text { for } 0 \leq \theta \leq \psi^{*},
$$

where $\psi^{*}$ is the expression $\left(3.1^{\prime}\right)$ with asterisks. The last inequality and (3.2) lead to the inequality

$$
\tan \alpha \geq \tan \alpha^{*}
$$

Now, from (3.27), (3.35), and (3.25) we find that 
(3.38) $\tan \alpha^{*}=\frac{2 \pi-\Omega^{*}}{\Omega^{*} \cot \beta^{\prime}+2 \pi \cot \phi^{*}}$

$$
\begin{aligned}
& =\frac{2 \pi-\frac{\theta^{\prime} \mu^{*}}{\tan (\alpha-\epsilon)+\mu^{*}}}{\frac{\theta^{\prime} \mu^{*}}{\tan (\alpha-\epsilon)+\mu^{*}} \cot \beta^{\prime}+\frac{2 \pi}{\tan (\alpha-\epsilon)+\mu^{*}}} \\
& =\frac{2 \pi \tan (\alpha-\epsilon)+\mu^{*}\left[2 \pi-\theta^{\prime}\right]}{2 \pi+\theta^{\prime} \mu^{*} \cot \beta^{\prime}} \\
& =\tan (\alpha-\epsilon)+\frac{\mu^{*}\left[2 \pi-\theta^{\prime}-\theta^{\prime} \tan (\alpha-\epsilon) \cot \beta^{\prime}\right]}{2 \pi+\theta^{\prime} \mu^{*} \cot \beta^{\prime}}
\end{aligned}
$$

Combining (3.37) with (3.38), and sending $\epsilon$ to zero, we get

$$
\tan \alpha \geq \tan \alpha+\mu_{0} \frac{2 \pi-\theta^{\prime} \frac{\tan \alpha+\tan \beta^{\prime}}{\tan \beta^{\prime}}}{2 \pi+\theta^{\prime} \mu_{0} \cot \beta^{\prime}}
$$

where, by (3.25), we have $\mu_{0}=\delta \% \Phi_{0}>0$, and where $\Phi_{0}$ is given by

$$
\Phi_{0}=\frac{2 \pi \tan \beta^{\prime}-\delta^{\prime}}{\tan \alpha+\tan \beta^{\prime}} .
$$

Since from (3.16) we also have

$$
\theta^{\prime}\left(\tan \alpha+\tan \beta^{\prime}\right)<2 \pi \tan \beta^{\prime},
$$

we find that the last term in (3.39) is positive. This, however, leads us to $\tan \alpha>\tan \alpha(0<\alpha<\pi / 2)$, an absurdity. Thus the assumption that (3.13) is not always an equality leads to a contradiction. This establishes the theorem.

4. Some further results. In Theorem 1 the existence only of the constants $\alpha$ and $\beta$ was proved. More careful analysis leads to the following more explicit result concerning the constants. Let $\gamma$ be a number such that $0<\gamma<\pi / 2$, and let $F_{\gamma}(u)$ be any interpolation function of $f(z)$ defined in the angle $|\arg u| \leq \gamma$. (Thus $F_{\gamma}(u)$ is of exponential type in the angle, verifying there $(3.32)$ and (3.33)). Then we have: 
(i) The unit circle is a cut for $f_{-1}(z)$ if, and only if, the positive axis is a direction of condensation of zeros for $F_{\gamma}(u)$. (That is, any angle $|\arg u| \leq \epsilon$ contains infinitely many zeros of $F_{\gamma}(u)$.)

(ii) If the positive axis is not a direction of condensation of zeros, then let the two numbers $\phi^{+}(\gamma)$ and $\phi^{-}(\gamma)$ be defined in the following way: $0<\phi^{+} \leq \gamma$ is such that $F_{\gamma}(u)$ has only a finite number of zeros in any angle $0 \leq \arg u \leq$ $\phi^{+}-\epsilon$, and infinitely many zeros in any angle $0 \leq \arg u \leq \phi^{+}+\epsilon_{0}$ (We put $\phi^{+}=\gamma$ if $F_{\gamma}(u)$ has a finite number of zeros in any angle $\left.0 \leq \arg u \leq \gamma-\epsilon_{0}\right)$ Similarly we define $\phi^{-}\left(0<\phi^{-} \leq \gamma\right)$ by the property that $F_{\gamma}(u)$ has a finite number of zeros in any angle $-\phi^{-}+\epsilon \leq \arg u \leq 0$ and infinitely many zeros in $-\phi^{-}-\epsilon \leq \arg u \leq 0$. Then, if $\phi^{+}<\gamma$, the constant $\alpha$ of Theorem 1 is the number $\phi^{+}$just defined. Similarly, if $\phi^{-}<\gamma$ we have $\beta=\phi^{-}$. Furthermore, if $\gamma_{n}$ is an increasing sequence such that $\gamma_{n} \rightarrow \pi / 2$, then we always have

$$
\alpha=\lim _{n=\infty} \phi^{+}\left(\gamma_{n}\right), \beta=\lim _{n=\infty} \phi^{-}\left(\gamma_{n}\right) .
$$

We shall omit here the proof of this result.

In Theorem 1 it was assumed that $f(z)$ is analytic in the whole plane cut along the line $1 \leq x<\infty$. Suppose now that we know only that $f(z)$ has the point $z=1$ as its only singularity on the unit circle, and that it is, furthermore, semi-isolated. That is, there exists $\rho>1$ such that $f(z)$ is analytic in the region bounded by the circle $|z|=\rho$ and the segment $1 \leq x \leq \rho$. It was shown by Pólya [4, p. 738] that in this case the singularities of $f(z)$ can be "separated" in the following way:

$$
f(z)=f^{*}(z)+f^{* *}(z)=\sum a_{n}^{*} a^{n}+\sum a_{n}^{* *} z^{n},
$$

where $f^{*}(z)$ is analytic in the whole plane cut along $1 \leq x<\infty$ while $f^{* *}(z)$ is analytic in the circle $|z|<\rho$. Obviously, we have $a_{n}=a_{n}^{*}+a_{n}^{* *}$ with

$$
\lim \sup \left|a_{n}^{* *}\right|^{1 / n}=\frac{1}{\rho} .
$$

Now, if the sequence $\left\{a_{n}\right\}$ satisfies (1.2), it is easily seen that $\left\{a_{n}^{*}\right\}$ also satisfies (1.2). There is also no loss of generality in assuming $a_{n}^{*} \neq 0$. From

$$
\frac{1}{a_{n}}=\frac{1}{a_{n}^{*}+a_{n}^{* *}}=\frac{1}{a_{n}^{*}}-\frac{a_{n}^{* *}}{a_{n} a_{n}^{*}},
$$


it follows that

$$
\lim \sup \left|\frac{1}{a_{n}}-\frac{1}{a_{n}^{*}}\right|^{1 / n} \leq \rho^{-1}
$$

Hence, if we put

$$
f_{-1}^{*}(z)=\sum \frac{1}{a_{n}^{*}} z^{n},
$$

we find that $f_{-1}(z)-f_{-1}^{*}(z)$ is analytic in $|z|<\rho$. Applying Theorem 1 to $f_{-1}^{*}(z)$, we arrive at the following conclusion:

THEOREM 2. Let $f(z)$ be analytic in the domain bounded by the circle $|z|<\rho(\rho>1)$ and the segment $1 \leq x \leq \rho$. Let (1.1) be the Taylor expansion of $f(z)$ in $|z|<1$, where $a_{n} \neq 0$ and where (1.2) is satisfied. Let $f_{-1}(z)$ be the "inverse" function defined by (1.1"). Then either the unit circle is a cut for $f_{-1}(z)$, or there exist two constants $\alpha$ and $\beta(0<\alpha \leq \pi / 2$ and $0<\beta \leq$ $\pi / 2)$, such that $f_{-1}(z)$ can be continued analytically along any ray te $e^{i \theta}, 0 \leq t<$ $r(\theta)$, where

$$
r(\theta)=\min \left\{e^{(\tan \alpha) \theta}, e^{(\tan \beta)(2 \pi-\theta)}, \rho\right\} \text { for } 0<\theta<2 \pi,
$$

and

$$
r(0)=1
$$

Furthermore, $r(\theta) e^{i \theta}$ is actually a singularity of $f_{-1}(z)$ if $r(\theta)<\rho$.

\section{REFERENCES}

1. L. Bieberbach, Lehrbuch der Funktionentheorie, vol. 2, Chelsea Publishing Company, 1945.

2. V. Bernstein, Lęcons sur les progrès récents de la théorie des séries de Dirichlet, Gauthier Villars, Paris, 1933.

3. J. Soula, Sur les points singuliers des deux fonctions $\sum a_{n} z^{n}$ et $\sum z^{n} / a_{n}$, Bull. Soc. Math. France 56 (1928), 33-49.

4. G. Pólya, Untersuchungen über Lücken und Singularitäten von Potenzreihen, Ann. of Math. 34 (1933), $731-777$.

5. E. C. Titchmarsh, The theory of functions, Oxford University Press, Oxford, Second Edition, 1939.

The Rice Institute 



\section{PACIFIC JOURNAL OF MATHEMATICS}

\section{EDITORS}

\section{R. M. RoBINSON \\ University of California Berkeley 4, California}

\author{
*R. P. Dilmorth \\ Califomia Institute of Technology \\ Pasadena 4, California
}

E. F. BeckenbaCh, Managing Editor

University of California

Los Angeles 24, California

${ }^{*}$ During the absence of Herbert Busemann in 1952.

\section{ASSOCIATE EDITORS}

$\begin{array}{llll}\text { R. P. DILWORTH } & \text { P. R. HALMOS } & \text { BØRGE JESSEN } & \text { J. J. STOKER } \\ \text { HERBERT FEDERER } & \text { HEINZ HOPF } & \text { PAUL LÉVY } & \text { E. G. STRAUS } \\ \text { MARSHALL HALL } & \text { R. D. JAMES } & \text { GEORGE PÓLYA } & \text { KÖSAKU YOSIDA }\end{array}$

\section{SPONSORS}

UNIVERSITY OF BRITISH COLUMBIA

CALIFORNIA INSTITUTE OF TECHNOLOGY

UNIVERSITY OF CALIFORNIA, BERKELEY

UNIVERSITY OF CALIFORNIA, DAVIS

UNIVERSITY OF CALIFORNIA, LOS ANGELES

UNIVERSITY OF CALIFORNIA, SANTA BARBARA

OREGON STATE COLLEGE

UNIVERSITY OF OREGON

\author{
UNIVERSITY OF SOUTHERN CALIFORNIA \\ STANFORD UNIVERSITY \\ WASHINGTON STATE COLLEGE \\ UNIVERSITY OF WASHINGTON \\ AMERICAN MATHEMATICAL SOCIETY \\ NATIONAL BUREAU OF STANDARDS, \\ INSTITUTE FOR NUMERICAL ANALYSIS
}

Mathematical papers intended for publication in the Pacific Journal of Miathematics should be typewritten (double spaced), and the author should keep a complete copy. Manuscripts may be sent to any of the editors. All other communications to the editors should be addressed to the managing editor, E. F. Beckenbach, at the address given above.

Authors are entitled to receive 100 free reprints of their published papers and may obtain additional copies at cost.

The Pacific Journal of Mathematics is published quarterly, in March, June, September, and December, by the University of California, Berkeley 4, California. The price per volume (4 numbers) is \$8.00; single issues, $\$ 2.50$. Special price to individual faculty members of supporting institutions and to individual members of the American Mathematical Society: $\$ 4.00$ per volume; single issues, $\$ 1.25$.

Subscriptions, orders for back numbers, and changes of address should be sent to the publishers, University of California Press, Berkeley 4, California.

Printed at Ann Arbor, Michigan. Entered as second class matter at the Post Office, Berkeley, California.

\section{UNIVERSITY OF CALIFORNIA PRESS • BERKELEY AND LOS ANGELES}




\section{Pacific Journal of Mathematics}

\section{Vol. 2, No. $4 \quad$ April, 1952}

Shmuel Agmon, On the singularities of Taylor series with reciprocal coefficients .................................... 431

Richard Arens, A generalization of normed rings ............... 455

Iacopo Barsotti, Intersection theory for cycles of an algebraic variety . . . . 473

Leonard M. Blumenthal, Two existence theorems for systems of linear inequalities ..................................... 523

Frank Herbert Brownell, III, Translation invariant measure over separable Hilbert space and other translation spaces................. 531

J. W. S. Cassels, On a paper of Niven and Zuckerman ............... 555

Nelson Dunford, Spectral theory. II. Resolutions of the identity .......... 559

Eugene Lukacs and Otto Szász, On analytic characteristic functions ...... 615

W. A. Mersman, Evaluation of an integral occurring in servomechanism theory.......................................... 627

Lawrence Edward Payne and Alexander Weinstein, Capacity, virtual mass, and generalized symmetrization......................... 633

Choy-Tak Taam, The boundedness of the solutions of a differential equation in the complex domain ...................................... 643 